\title{
Muscarinic cholinergic signaling and overactive bladder-like symptoms associated with invasive bladder cancer
}

\author{
WEI WEI $^{1 *}$, MINGGANG WANG $^{2 *}$, YUNGLONG $^{3}{ }^{3}$, QINGGUI MENG $^{1}$,

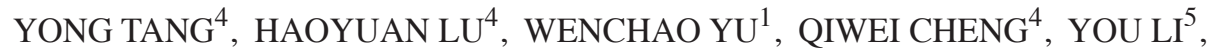 \\ LONG XU ${ }^{5}$, SHAOJUN JIAN ${ }^{6}$, YUEXIAN WU ${ }^{7}$, XIANLIN YI ${ }^{4,5}$ and KEJI XIE ${ }^{6}$ \\ ${ }^{1}$ Department of Radiology, Affiliated Tumor Hospital of Guangxi Medical University, Nanning, \\ Guangxi 530021; ${ }^{2}$ Department of Urology, Affiliated Hospital of Qinghai University, Xining, Qinghai 810016; \\ ${ }^{3}$ Department of Urology, First People's Hospital of Kunshan, Kunshan, Jiangsu 215300; ${ }^{4}$ Department of Urology, \\ Affiliated Tumor Hospital of Guangxi Medical University, Nanning, Guangxi 530021; ${ }^{5}$ Hubei Engineering Laboratory \\ for Synthetic Microbiology, Wuhan Institute of Biotechnology, Wuhan, Hubei 430075; ${ }^{6}$ Department of Urology, \\ Guangzhou First People's Hospital, Guangzhou Medical University, Guangzhou, Guangdong 510180; ${ }^{7}$ Department \\ of Respiratory, Nanfang Hospital of Southern Medical University, Guangzhou, Guangdong 510515, P.R. China
}

Received April 6, 2017; Accepted March 16, 2018

DOI: $10.3892 / \mathrm{ol} .2018 .8715$

\begin{abstract}
The objective of the present study was to explore the association between muscarinic cholinergic signaling and urothelial bladder tumors. Possible associations among overactive bladder (OAB) symptoms and bladder tumors were retrospectively investigated using a multicenter Chinese database with prospectively collected data since 2010. Firstly, it was demonstrated that OAB symptoms, such as urgency, were more severe in patients with invasive bladder cancer and were associated with a reduced prognosis. Following this, muscarinic cholinergic receptor 3 (M3R) expression in urothelium was determined to be lower in invasive cancer tissue than in adjacent non-cancerous tissue, yet M3R upregulation was associated with a reduced progression free survival (PFS) time. Additionally, it was also demonstrated that muscarinic cholinergic receptor 2 (M2R) was upregulated in the sub-urothelium, and this was also associated with a reduced PFS time. Furthermore, it was determined that cholinesterase and acetylcholinesterase were lower in invasive cancer than in non-invasive cancer. In conclusion,
\end{abstract}

Correspondence to: Dr Xianlin Yi, Department of Urology, Affiliated Tumor Hospital of Guangxi Medical University, 71 Hedi Road, Nanning, Guangxi 530021, P.R. China

E-mail:yzztx@126.com

Dr Keji Xie, Department of Urology, Guangzhou First People's Hospital, Guangzhou Medical University, Guangzhou, Guangdong 510180, P.R. China

E-mail: xiekeji@sina.com

*Contributed equally

Key words: bladder cancer, muscarinic receptor, overactive bladder the results indicated that $\mathrm{M} 3 \mathrm{R}$ expression was downregulated in invasive bladder cancer, which may have a role as a protective anti-oncogene, in contrast to its oncogenic role in numerous other cancer types. Therefore, muscarinic cholinergic signaling may be a novel therapeutic target for treating bladder cancer.

\section{Introduction}

Bladder cancer is the fourth most common tumor among males in the United States of America (1). The most frequent symptom of bladder cancer is hematuria, whilst urgency, dysuria and bladder irritation indicate muscle-invasive bladder tumors or carcinoma in situ (cis) (2). A problem for the diagnosis of bladder cancer is that patients with stage $T_{a}$ or $T_{1}$ disease may not manifest any of these symptoms (1-3).

Numerous patients with bladder diseases may exhibit a complex of symptoms known as overactive bladder (OAB), characterized by urgency, frequency and nocturia, with or without urinary incontinence (4). It was reported that metastatic breast cancer was also associated with OAB-like symptoms $(4,5)$. Whether these symptoms are associated with molecular features or with prognosis of bladder cancer remains unclear. These data may be beneficial for understanding the onset and progression of bladder cancer.

Antagonists of muscarinic acetylcholine receptors (mAChRs) remain the first-line therapy for treating OAB symptoms, such as urgency (6). Understanding the role of $\mathrm{mAChRs}$ in OAB requires the examination of all five receptor subtypes (M1R-M5R), all of which being located in the urothelium (4). The urothelium could respond to muscarinic agonist stimulation by self-releasing acetylcholine (ACh), which may modulate afferent activity in turn (7). The urothelium assists in filling and distension of the bladder, and it protects the underlying stroma (8). Of non-muscle invasive bladder cancer, $70 \%$ were confined to the urothelium (5). 
Muscarinic cholinergic receptor 3 (M3R) is one of the major receptors associated with micturition in normal human bladder and OAB (9); however, M3R transcripts have not been detected in the J82, RT4, T24 and 5,637 urothelial cancer cell lines (10). Recently, a study also determined that M3R expression was decreased in the bladder cancer group, compared with non-cancer control individuals (9). Nevertheless, the clinical features were not mentioned in these studies, and invasive bladder cancer was not focused on $(9,10)$.

MAChRs are expressed in several tumor types, including colon, ovary, lung, breast and prostate cancer, and the genes encoding M1R and M3R have been demonstrated to function as conditional oncogenes (11), activating downstream phosphatidylinositol hydrolysis to induce cell proliferation (12). In addition to clarifying the potential role of MAChRs in bladder cancer, studies are required to explore the potential role of muscarinic cholinergic signaling. Such signaling serves an important role in colon cancer (13), but whether it also participates in bladder cancer is unclear. This signaling can be partially measured via assays for several enzymes involved in auto- and paracrine signaling, including acetylcholinesterase (AChE), butyrylcholinesterase and choline acetyltransferase (ChAT) (14). Muscarinic signaling in the urothelium also contributes to OAB-like symptoms $(4,15)$.

To begin to address these gaps in the literature, data that had been prospectively entered into a multicenter database, of patients with invasive bladder cancer in China, were retrospectively analyzed. Possible associations between OAB-like symptoms and muscarinic cholinergic signaling were examined using this data.

\section{Patients and methods}

Study design and ethical approval. A multicenter database between January 2010 to December 2015 was established at the Tumor Hospital of Guangxi Medical University (Nanning, China), Nanfang Hospital of Southern Medical University (Guangzhou, China) and Guangzhou First People's Hospital (Guangzhou, China), containing medical records of patients with OAB, suspected or diagnosed bladder tumors since 2010. The patients written informed consent were obtained at the time of data collection. The mean age was 58.2 (7-85) years, 203 were male and 71 were female. The present study was approved by the ethics committee of institutes aforementioned. Information on OAB-like symptoms were recorded prospectively in this database, after which the patients underwent bladder biopsy, transurethral resection or radical cystectomy. Overall OAB symptom severity was assessed using the OAB symptom score (OABSS) (15). Investigators collected symptom data prior to definitive diagnosis or pathology results became available.

Inclusion criteria and exclusion criteria. Patients in the database who were confirmed to have bladder tumors based on bladder biopsy, transurethral resection or radical cystectomy between January 2010 and December 2015 were eligible. Patients were excluded from the study if they had previously been diagnosed with OAB or interstitial cystitis or if they had a history of severe urinary system infection, pelvic radiotherapy and sexually transmitted diseases.
Clinical outcomes. Outcomes were time to recurrence, progression free survival (PFS) and overall survival. Time to recurrence and PFS were calculated as the date from radical cystectomy to the first documented recurrence. A single patient was diagnosed with cis, a type of superficial bladder tumor which is not muscle-invasive, and was not included in the PFS observation.

Reverse transcription-polymerase chain reaction (RT-PCR) analysis of MAChRs M1R-M5R. Bladder mucosa specimens were obtained by cystoscopy from 10 patients with OAB, and total RNA was extracted using TRIzol ${ }^{\circledR}$ reagent (Invitrogen; Thermo Fisher Scientific, Inc., Waltham, MA, USA), according to the manufacturer's instructions. Expression levels of M1R-M5R transcripts were determined by RT-PCR as previously described (16). Total RNA $(1.5 \mu \mathrm{g})$ was reverse transcribed for $60 \mathrm{~min}$ at $37^{\circ} \mathrm{C}$ using random primers from the M-MLV First Strand kit (Invitrogen; Thermo Fisher Scientific, Inc.). PCR amplification was performed for $2 \mathrm{~min}$ at $50^{\circ} \mathrm{C}$, then $10 \mathrm{~min}$ at $95^{\circ} \mathrm{C}$, followed by 45 cycles of $15 \mathrm{sec}$ at $95^{\circ} \mathrm{C}$ and $60 \mathrm{sec}$ at $58^{\circ} \mathrm{C}$.

Histopathology and immunohistochemistry against $M 1 R-M 5 R$. Polyclonal antibodies against M1R-M5R were purchased from Abcam (Cambridge, UK), and DAB substrate liquid was obtained from Sigma-Aldrich (Merck KGaA, Darmstadt, Germany). A total of $4 \mu \mathrm{m}$-thick sections from tumor tissue and tumor-adjacent tissue were embedded in paraffin, fixed in $4 \%$ paraformaldehyde for $24 \mathrm{~h}$ at room temperature, deparaffinized, subjected to antigen retrieval (citrate buffer for $30 \mathrm{~min}$ at $100^{\circ} \mathrm{C}$; rehydration in a descending ethanol series at 100, 95, 85 and $75 \%$ for $10 \mathrm{~min}$ ), incubated with $3 \%$ hydrogen peroxide for $10 \mathrm{~min}$ at room temperature and mounted on slides. Slides were incubated with rabbit antibodies to CHRM1 (1:1,000; Abcam; cat. no. ab180636), CHRM2 (1:500; Abcam; cat. no. ab188891), CHRM3 (1:100; Abcam, ab150480), CHRM4 (1:1,000; Abcam, ab150518), CHRM5 antibody (1:1,000; Abcam; cat. no. ab150531) overnight at $4^{\circ} \mathrm{C}$. The following day, these tissues were washed with PBS three times, and goat anti-rabbit immunoglobulin G horseradish peroxidase-conjugated (1:1,000; cat. no. HA1001; HuaBio Inc., Cambridge, MA, USA) secondary antibody incubated for $1 \mathrm{~h}$ at room temperature. DAB substrate liquid was used for the slides and visualized under a light microscope (magnification, x100; cat. no. CX31-LV320; Olympus Corporation, Tokyo, Japan).

Serum levels of cholinesterase (ChE). After patients had been hospitalized for 2 days, venous blood $(2 \mathrm{ml})$ was collected on an empty stomach, serum was isolated by centrifugation for $10 \mathrm{~min}$ at $1,509 \mathrm{x} \mathrm{g}$ at room temperature. Levels of $\mathrm{ChE}$ in serum were assayed using the cholinesterase kit (CUSABIO, Wuhan, China) and conducted according to the manufacturer's protocol.

Statistical analysis. Data were plotted using GraphPad Prism 5 (GraphPad Software, Inc., La Jolla, CA, USA) and presented as the mean \pm the standard deviation. Statistical tests were applied within SPSS 21 (IBM Corp., Armonk, NY, USA). P<0.05 was considered to indicate a statistically significant difference, and 
all reported P-values were two-sided. Differences between patient groups were assessed for significance using Student's t-test, including comparing serum levels of $\mathrm{ChE}$ and baseline information. Differences in M1R-M5R expression between muscle-invasive non-muscle-invasive bladder cancer were analyzed using one-way analysis of variance followed by the Student-Newman-Keuls post hoc test. When assessing differences in skewed data using multiple comparisons, the non-parametric test was used. For Table II, Kruskal-Wallis test was used to compare three groups, whilst the Mann-Whitney $\mathrm{U}$ test was used to compare two groups.

Among 26 patients who underwent radical cystectomy, PFS was estimated using Kaplan-Meier plots with a log rank test and multivariate Cox proportional hazards regression.

\section{Results}

Differences in OAB-like symptoms between muscle-invasive and non-muscle-invasive bladder cancers. The 228 patients included in the retrospective analysis were classified as having invasive or non-invasive bladder cancer (Table I). Incidence and severity of OAB-like symptoms, including urgency, daytime frequency and nocturia was higher in the invasive group, compared with the non-invasive group (Table II). The OAB-like symptoms in patients with malignant tumors were more severe, compared with those with benign tumors ( $\mathrm{P}=0.00001$; Table II).

The core symptom of OAB is urgency (4). Among 26 patients who underwent radical cystectomy, those experiencing 'daily $\geq 1$ ' urgency indicated lower PFS, compared with those with less severe urgency ( $\mathrm{P}=0.04962$; Fig. 1A). Cox regression identified urgency as an independent predictor of PFS following radical cystectomy.

M3R expression in urothelium and PFS in patients undergoing radical cystectomy. M3R expression was also observed in PFS study. Higher M3R expression in the urothelium of the 26 patients with muscle-invasive bladder cancer who underwent radical resection was associated with reduced PFS $\left[\chi^{2}=8.811\right.$, degrees of freedom $(\mathrm{df})=3, \mathrm{P}=0.032$; Fig. 1B]. Similar results were obtained when M3R expression in the sub-urothelium was analyzed $\left(\chi^{2}=11.651, \mathrm{df}=3, \mathrm{P}=0.009\right)$.

Differences in M1R-M5R expression between muscle-invasive and non-muscle-invasive bladder cancers. Expression levels of M3R transcript in bladder mucosa was significantly higher in patients with $\mathrm{OAB}$, compared with the normal group $(\mathrm{P}=0.00088$; Fig. 1C). Among patients with bladder cancer, M3R staining intensity in immunostained bladder urothelium was lower in the muscle-invasive group, compared with the non-muscle-invasive group (Fig. 1D). M3R staining intensity in the sub-urothelium, as well as the mortality rate, was similar between the two patient subgroups (Data not shown).

Downregulation of M3R in urothelium of patients with bladder cancer. M3R expression in bladder urothelium (Fig. 2A), sub-urothelium (Fig. 2B) and detrusor (Fig. 2C) were determined in patients with bladder cancer. M3R expression in bladder urothelium was lower in patients with bladder cancer, whether muscle-invasive or invasive,
Table I. Baseline characteristics of 228 Chinese patients with bladder tumor.

\begin{tabular}{lc}
\hline Variable & Value $^{\mathrm{a}}$ \\
\hline Age, years & \\
Mean \pm SE & $0.5 \pm 13.53$ \\
Median & 61 \\
Interquartile range & $50-71$
\end{tabular}

Sex

Male

$181(79.4)$

Female

Previous operation

No

TUR

Open (partial cystectomy)

Previous intravesical instillation

No

$183(80.3)$

Yes

Total bladder cancer $(n=206)$

Non-muscle-invasive

$109(47.8)$

Muscle-invasive

Total bladder cancer $(n=206)$

Non-invasive

Invasive

Grade of bladder cancer (WHO 2004, n=199)

PUNLMP

$10(4.4)$

Low-grade

High-grade

99 (43.4)

$\mathrm{N}$ stage of bladder cancer $(\mathrm{n}=206)$

N0

$175(76.7)$

N1

$\mathrm{N} 2$

N3

Metastasis of bladder cancer $(n=206)$

No

$198(86.8)$

Yes

Pathology

PUNLMP

Ta

T1

$\mathrm{T} 2 \mathrm{a}$

$\mathrm{T} 2 \mathrm{~b}$

$19(8.3)$

T3a

$14(6.1)$

$13(5.7)$

$11(4.8)$

$\mathrm{T} 4 \mathrm{a}$

$10(4.4)$

$1(0.4)$

Tis

$22(9.6)$

Benign tumor ${ }^{\mathrm{b}}$

PUNLMP, papillary urothelial neoplasm of low malignant potential; TUR, transurethral resection; WHO, World Health Organization; SE, standard error. Mitomycin, pirarubicin and gemcitabine were used in previous intravesical instillation. ${ }^{a} \mathrm{n}(\%)$, except for age. ${ }^{b}$ Benign tumor is constituted of inverted papilloma and cystitis glandularis. 
Table II. Differences in overactive bladder-like symptoms between benign tumor, invasive and non-invasive bladder cancer.

\begin{tabular}{|c|c|c|c|c|}
\hline Factor & $\operatorname{Benign}^{a}(n=11)$ & Non-invasive $(n=55)$ & Invasive $(n=62)$ & P-value \\
\hline No. urgency (\%) & & & & $0.0017^{\mathrm{b}}$ \\
\hline No urgency & $2(18.18)$ & $29(52.73)$ & $15(24.19)$ & \\
\hline Urgency $\geq 1 /$ week & $1(9.09)$ & $2(3.64)$ & $3(4.84)$ & \\
\hline Urgency=1/day & $2(18.18)$ & $4(7.27)$ & $4(6.45)$ & \\
\hline Urgency $=2-4 /$ day & $2(18.18)$ & $11(20)$ & $15(24.19)$ & \\
\hline Urgency $\geq 5 /$ day & $4(36.36)$ & $9(16.36)$ & $25(40.32)$ & \\
\hline No. daytime frequency (\%) & & & & $0.0322^{\mathrm{b}}$ \\
\hline$\leq 7$ & $3(27.27)$ & $28(50.91)$ & $23(37.10)$ & \\
\hline $8-14$ & $5(45.45)$ & $24(43.64)$ & $23(37.10)$ & \\
\hline$\geq 15$ & $3(27.27)$ & $3(5.45)$ & $16(25.81)$ & \\
\hline No. nighttime frequency $(\%)$ & & & & $0.0001^{\mathrm{b}}$ \\
\hline 0 & $2(18.18)$ & $4(7.27)$ & $2(3.23)$ & \\
\hline 1 & $1(9.09)$ & $12(21.82)$ & $2(3.23)$ & \\
\hline 2 & $4(36.36)$ & $18(32.73)$ & $12(19.35)$ & \\
\hline$\geq 3$ & $4(36.36)$ & $21(38.19)$ & $46(74.19)$ & \\
\hline No. OABSS $(\%)$ & & & & $0.0016^{\mathrm{b}}$ \\
\hline Urgency $<2$ & $0(0)$ & $29(52.73)$ & $15(24.19)$ & \\
\hline Mild & $6(54.55)$ & $4(7.27)$ & $3(4.84)$ & \\
\hline Moderate & $2(18.18)$ & $18(32.73)$ & $33(53.23)$ & \\
\hline Severe & $3(27.27)$ & $4(7.27)$ & $11(17.74)$ & \\
\hline
\end{tabular}

\begin{tabular}{|c|c|c|c|}
\hline Factor & Non-muscle-invasive $(\mathrm{n}=55)$ & Muscle-invasive $(\mathrm{n}=62)$ & \\
\hline No. urgency (\%) & & & $0.0005^{\mathrm{c}}$ \\
\hline No urgency & $29(52.70)$ & $15(24.20)$ & \\
\hline Urgency $\geq 1 /$ week & $2(3.60)$ & $3(4.80)$ & \\
\hline Urgency=1/day & $4(7.30)$ & $4(6.50)$ & \\
\hline Urgency $=2-4 /$ day & $11(20)$ & $15(24.20)$ & \\
\hline Urgency $\geq 5 /$ day & $9(16.40)$ & $25(40.30)$ & \\
\hline No. daytime frequency (\%) & & & $0.0198^{b}$ \\
\hline$\leq 7$ & $28(50.90)$ & $23(37.10)$ & \\
\hline $8-14$ & $24(43.60)$ & $23(37.10)$ & \\
\hline$\geq 15$ & $3(5.50)$ & $16(25.80)$ & \\
\hline No. nighttime frequency $(\%)$ & & & $<0.0001^{\mathrm{b}}$ \\
\hline 0 & $4(7.30)$ & $2(3.20)$ & \\
\hline 1 & $12(21.80)$ & $2(3.20)$ & \\
\hline 2 & $18(32.70)$ & $12(19.40)$ & \\
\hline$\geq 3$ & $21(38.20)$ & $46(74.20)$ & \\
\hline No. OABSS (\%) & & & $0.0191^{\mathrm{b}}$ \\
\hline Urgency<2 & $29(52.70)$ & $15(24.20)$ & \\
\hline Mild & $4(7.30)$ & $3(4.80)$ & \\
\hline Moderate & $18(32.70)$ & $33(53.20)$ & \\
\hline Severe & $4(7.30)$ & $11(17.70)$ & \\
\hline
\end{tabular}

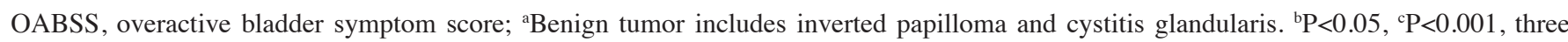
groups were compared using Kruskal-Wallis test and two groups were compared using Mann-Whitney U test.

compared with the non-muscle-invasive or non-invasive group, respectively (Fig. 2D-G). For patients with invasive cancer, M3R expression in the urothelium demonstrated a decreasing gradient in the following order: Adjacent tissue 
A

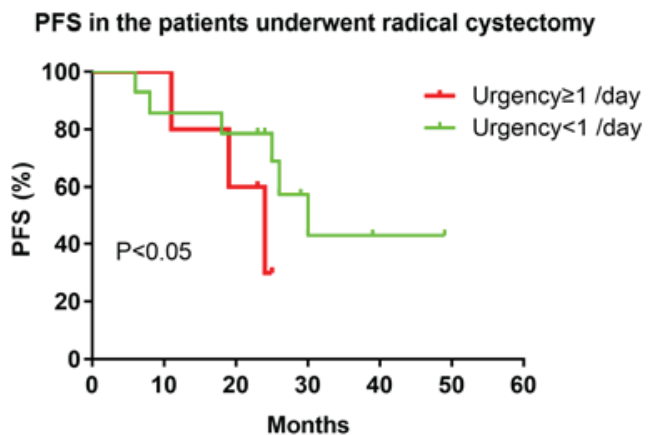

C

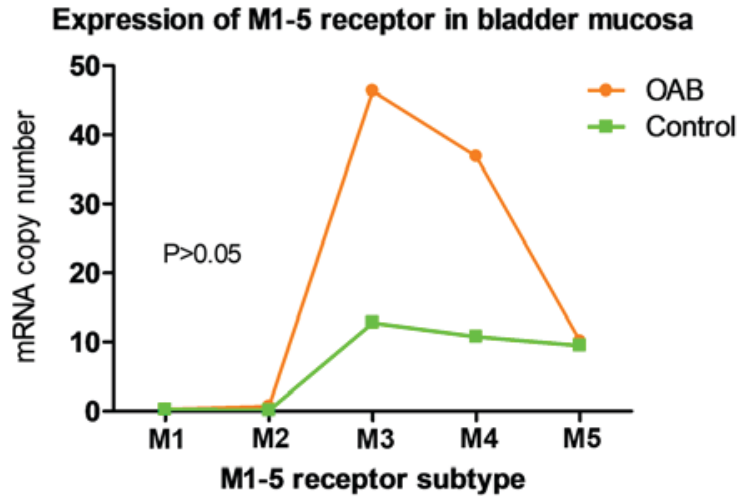

B M3 of urothelium in patients who underwent radical cystectomy

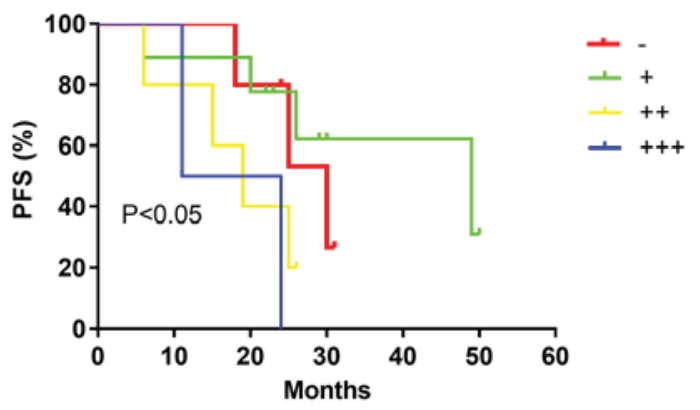

D

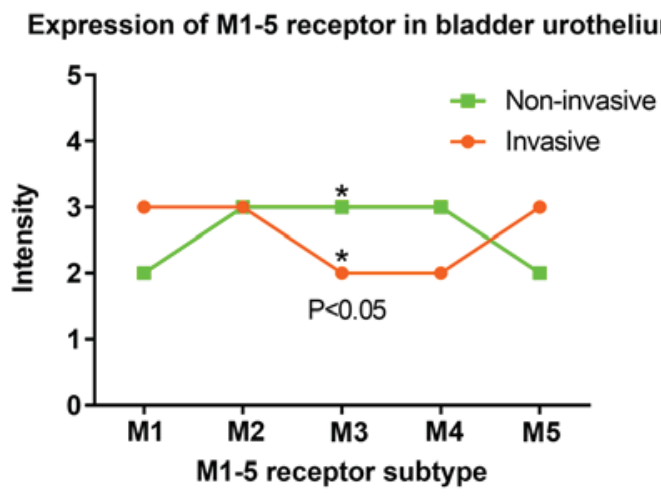

Figure 1. Expression of M1-5R of urothelium in OAB and bladder cancer and urgency in PFS. (A) Patients experiencing 'daily $\geq 1$ ' urgency demonstrated significantly lower PFS, compared with those experiencing 'daily $<1$ ' $(\mathrm{P}=0.0496)$. (B) $\mathrm{PFS}$ in patients with invasive bladder cancer who underwent radical cystectomy. M3R expression was negatively associated with PFS ( $\mathrm{P}=0.032$ ). (C) Expression levels of mAChRs M1-5R in bladder mucosa of patients with $\mathrm{OAB}$ based on reverse transcription-polymerase chain reaction. (OAB group, M1-5, $\mathrm{n}=10,9,10,10,10$; Control group, $\mathrm{M} 1-5, \mathrm{n}=11,9,11,11,11 ; \mathrm{P}=0.56,0.22$, $0.56,0.43,0.92)$. (D) M1-5R proteins in bladder urothelium of patients with bladder cancer based on immunohistochemistry. M3R staining ( $\mathrm{P}<0.05)$ was less intense in patients with muscle-invasive bladder cancer, even though this group had more severe OAB-like symptoms. PFS, progression free survival; M1-5R, muscarinic cholinergic receptor 1-5; OAB, overactive bladder.

lying $>2 \mathrm{~cm}$ from the tumor; adjacent tissue lying $0.5-1 \mathrm{~cm}$ from the tumor; tumor tissue (Fig. 2H). M3R expression of detrusor was higher in the muscle-invasive and invasive groups (Fig. 2I). When patients were classified as low or high-grade, according to WHO 2004 criteria (17), M3R expression in detrusor muscle was significantly higher in the low-grade subgroup $\left(\chi^{2}=7.348, P=0.046\right)$.

Upregulation of $M 2 R$ in urothelium of patients with bladder cancer. Expression levels of M2R in urothelium were significantly increased in patients with invasive bladder cancer. In the sub-urothelium, M2R expression was significantly higher in the invasive group, compared with the non-invasive group $\left(\chi^{2}=10.274, \mathrm{df}=3, \mathrm{P}=0.016\right)$, and significantly higher in the muscle-invasive group, compared with the non-muscle-invasive group $\left(\chi^{2}=8.289, \mathrm{df}=3, \mathrm{P}=0.038\right)$. Among the 26 patients who underwent radical cystectomy, the level of M2R expression in the sub-urothelium negatively associated with PFS $\left(\chi^{2}=14.276, \mathrm{df}=3, \mathrm{P}=0.003\right)$.

Expression MIR and M4R in patients with bladder cancer. M1R expression in the sub-urothelium was significantly higher in the invasive group $\left(\chi^{2}=9.151, \mathrm{P}=0.027\right)$ and muscle-invasive group $\left(\chi^{2}=10.028, \mathrm{P}=0.034\right)$, compared with the non-invasive and non-muscle-invasive group. Among patients with muscle-invasive cancer, M1R expression in urothelium was significantly lower in tumor, compared with in adjacent tissues $\left(n=23, \chi^{2}=8.982, P=0.01\right.$; Fig. 3$)$.
In addition, M1R expression in urothelium of patients with invasive cancer negatively associated with tumor $\mathrm{T}$ stage $\left(\chi^{2}=24.73, \mathrm{P}=0.029\right)$.

$\mathrm{M} 4 \mathrm{R}$ expression in the sub-urothelium was significantly higher in the muscle-invasive group, compared with the non-muscle-invasive group $\left(\chi^{2}=7.770, \mathrm{P}=0.049\right)$. M4R expression in invasive sub-urothelial tissue negatively associated with tumor T stage $\left(\chi^{2}=29.472, P=0.009\right)$. An increasing gradient of M4R expression in urothelium also existed in the following order: Adjacent tissue lying $>2 \mathrm{~cm}$ from the tumor; adjacent tissue lying $0.5-1 \mathrm{~cm}$ from the tumor; and tumor tissue (Fig. 3).

Other indicators of cholinergic signaling in bladder cancer. Serum levels of $\mathrm{ChE}$ were significantly lower in patients with invasive cancer $(\mathrm{n}=85,7614.69 \pm 2317.75 \mathrm{U} / \mathrm{l})$, compared with those with non-invasive cancer ( $\mathrm{n}=108,8570.86 \pm 2037.64 \mathrm{U} / \mathrm{l})$ $(\mathrm{t}=2.999, \mathrm{P}=0.003)$. Similar results were obtained when patients with muscle-invasive and non-muscle-invasive cancer $(\mathrm{t}=2.955, \mathrm{P}=0.004)$, or patients with low and high-grade cancer $(\mathrm{t}=3.546, \mathrm{P}=0.001)$ were compared.

AChE levels in urothelium and sub-urothelium were similar between patients with invasive or non-invasive cancer. Among those patients with muscle-invasive cancer who underwent radical cystectomy, $\mathrm{AChE}$ expression levels in the urothelium were positively associated with PFS. Expression levels of AChE within the detrusor demonstrated a negative gradient: Adjacent tissue lying $>2 \mathrm{~cm}$ from the tumor; tumor 

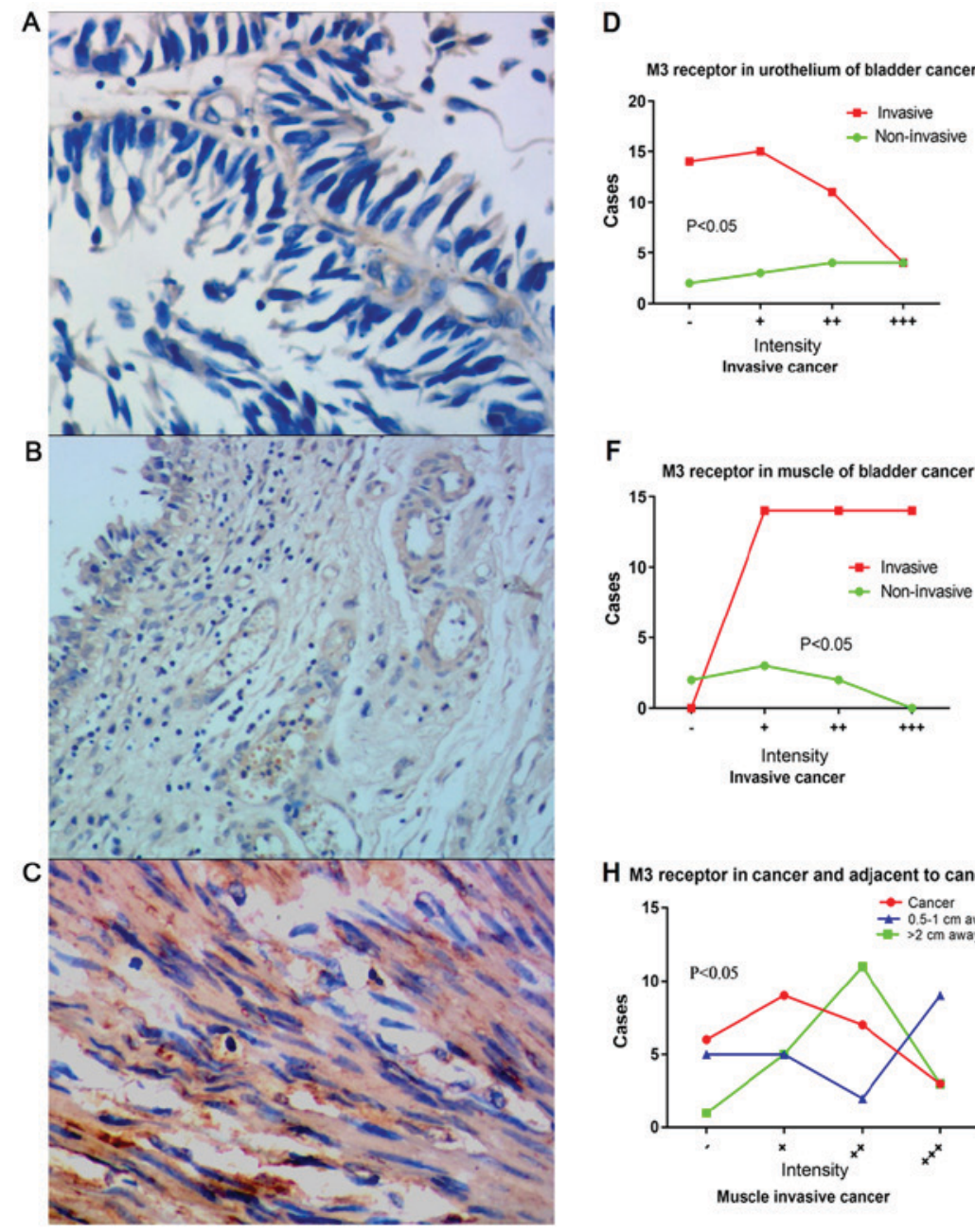

E
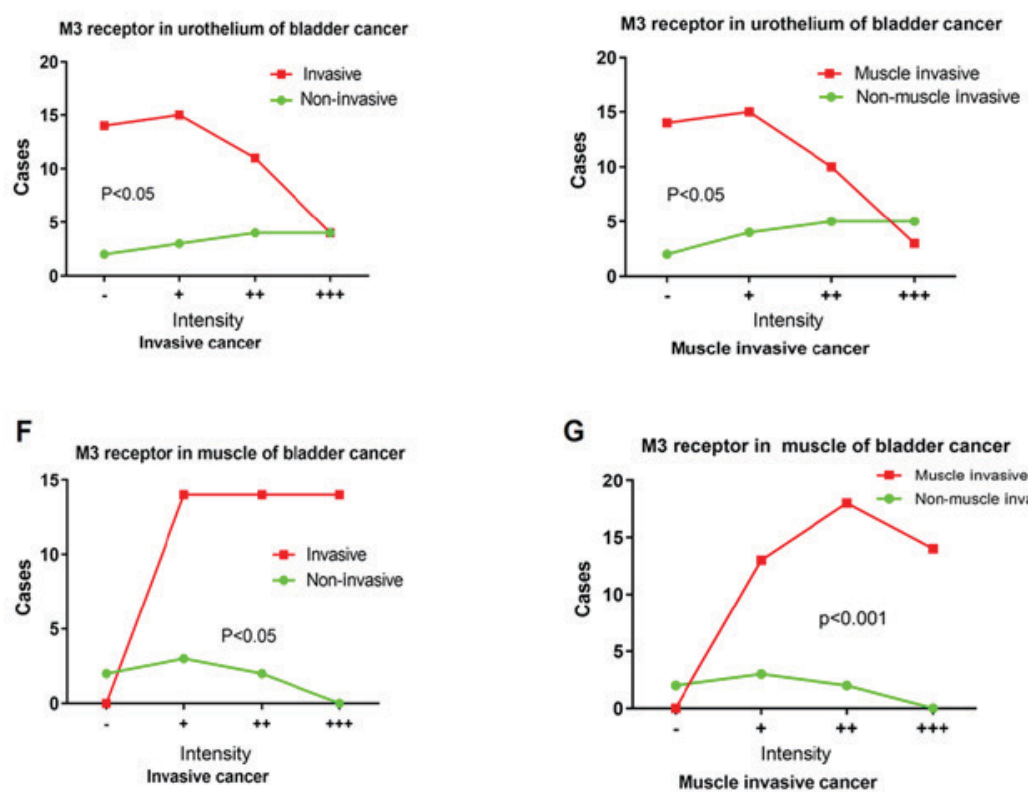

G

G M3 receptor in muscle of bladder cancer

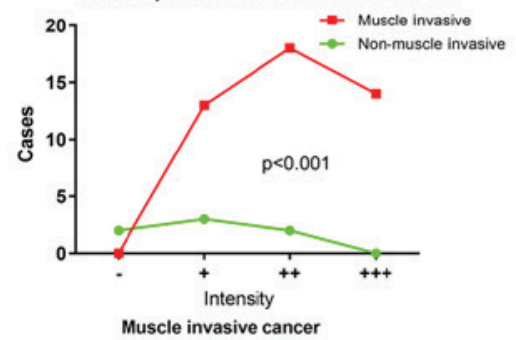

H M3 receptor in cancer and adjacent to cancer tissue

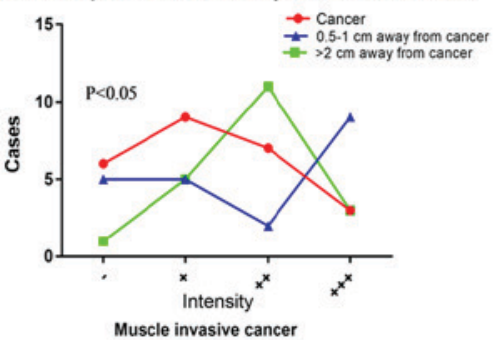

I M3 receptor in muscle of bladder cancer

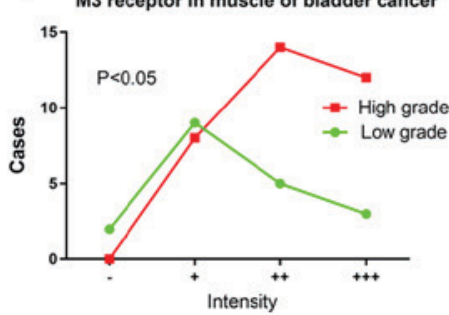

Figure 2. Expression of M3R in bladder cancer tissue. Representative immunostained photomicrographs indicating M3R expression in (A) bladder urothelium, (B) sub-urothelium and (C) detrusor of patients with bladder cancer. Differences in M3R staining intensity of urothelium between (D) invasive and non-invasive bladder cancer tissues and (E) muscle invasive and non-muscle invasive bladder cancer tissues ( $\mathrm{P}=0.038,0.017)$. Differences in M3R staining intensity of detrusor between $(\mathrm{F})$ invasive and non-invasive bladder cancer tissues and $(\mathrm{G})$ muscle invasive and non-muscle invasive bladder cancer tissues $(\mathrm{P}=0.001,0.001)$. (H) Differences in M3R staining intensity between tumor urothelium tissue and adjacent tissue ( $\mathrm{P}=0.023)$. (I) Differences in M3R staining intensity in detrusor between patients with low-grades and high-grade bladder cancer $(\mathrm{P}=0.046)$. M3R, muscarinic cholinergic receptor 3 .

tissue; adjacent tissue lying $0.5-1 \mathrm{~cm}$ from the tumor $\left(\chi^{2}=8.486\right.$, $\mathrm{df}=2, \mathrm{P}=0.014)$.

Among muscle-invasive cancer tissues, rate of ChAT expression based on immunostaining of urothelium indicated a negative gradient: Tumor tissue; adjacent tissue lying $>2 \mathrm{~cm}$ from the tumor; adjacent tissue lying $0.5-1 \mathrm{~cm}$ from the tumor. Furthermore, the expression of matrix metalloproteinase- 9 (MMP-9) in urothelium demonstrated an increasing gradient in the following order: Adjacent tissue lying $>2 \mathrm{~cm}$ from the tumor; adjacent tissue lying $0.5-1 \mathrm{~cm}$ from the tumor; and tumor tissue (Fig. 3).

\section{Discussion}

The present study indicated that urgency and other OAB-like symptoms were more serious in patients with muscle-invasive bladder cancer than in those with non-muscle-invasive cancer, based on daytime frequency, nocturia, urgency and OABSS. Patients with invasive or muscle-invasive cancer manifested more severe OAB-like symptoms than patients with benign bladder tumors. The results provided the first evidence that urgency was more severe in invasive bladder cancer than in non-invasive cancer, and is associated with reduce prognosis following radical cystectomy.

Bladder carcinoma invading the detrusor could produce secondary urgency and frequency. However, it was determined that the differences existed in invasive or muscle invasive cancers. This may be because the invasive bladder cancer only invaded the lamina propria but not the detrusor.

All five subtypes of mAChRs (M1R-M5R) in the patient tissue were detected, consistent with work in normal urothelium (4); however, it was determined that expression of M3R in the urothelium of patients with invasive bladder cancer was much lower than that of other receptors (Fig. 1D), consistent with reports of M3R was decreased in bladder cancer tissues and a gradient of $\mathrm{M} 1 \geq \mathrm{M} 2>\mathrm{M} 3$ in $\mathrm{T} 24$ bladder cancer cells $(18,19)$, but different from a gradient of $\mathrm{M} 3>\mathrm{M} 4>\mathrm{M} 5>\mathrm{M} 2>\mathrm{M} 1$ in a study of patients with OAB (17). The current results also contrast with the gradient of $\mathrm{M} 2>\mathrm{M} 3=\mathrm{M} 5>\mathrm{M} 4=\mathrm{M} 1$ in normal human urothelium (Fig. 1C) (20).

M3R serves an important role in OAB (21). The M3R expression was higher in bladder mucosa of patients with $\mathrm{OAB}$, 
A
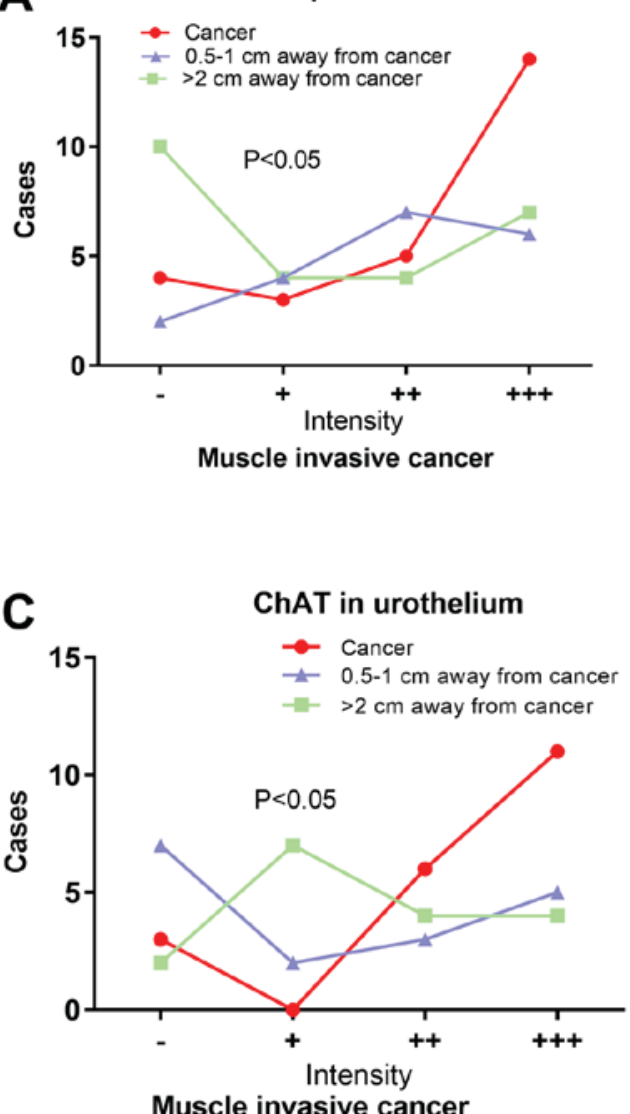

B

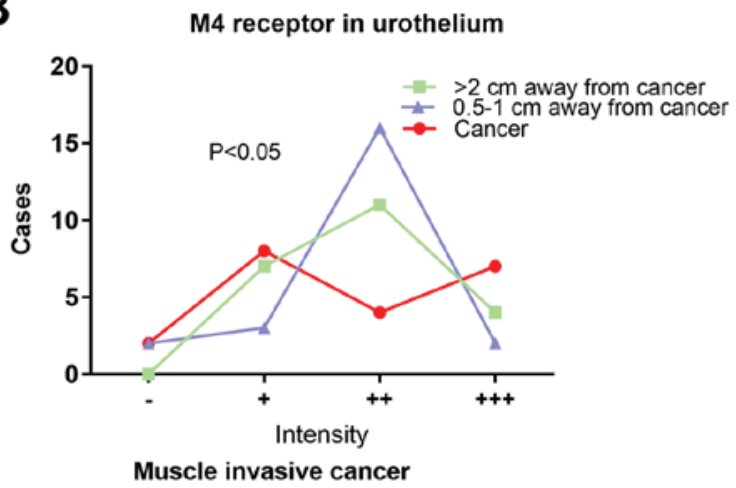

D

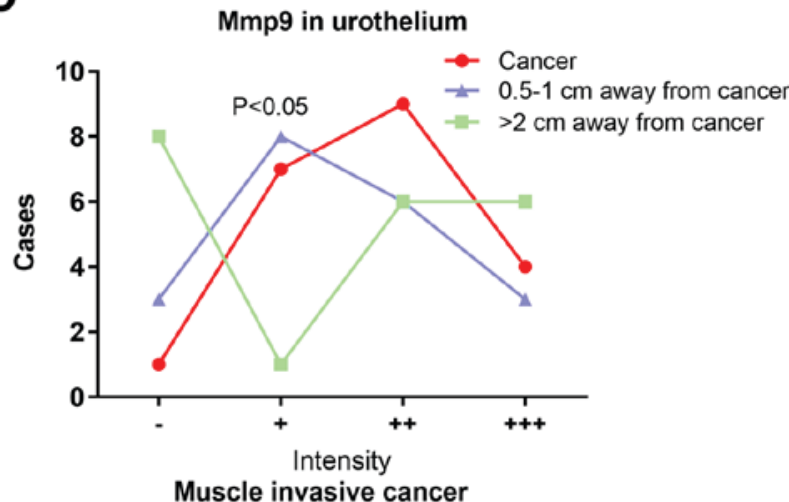

Figure 3. Other indicators of cholinergic signaling in bladder cancer. Differences in proportions of urothelial cells positive for (A) $\mathrm{M} 1 \mathrm{R}$ ( $\mathrm{P}=0.010)$, (B) $\mathrm{M} 4 \mathrm{R}$ $(\mathrm{P}=0.010),(\mathrm{C})$ ChAT $(\mathrm{P}=0.033)$ and $(\mathrm{D}) \mathrm{MMP}-9(\mathrm{P}=0.009)$ between bladder cancer tissue and adjacent tissue. MMP-9, matrix metalloproteinase-9; ChAT, Choline acetyltransferase; M1R, muscarinic cholinergic receptor 1; M4R, muscarinic cholinergic receptor 4.

compared with the normal group (17). M3R upregulation was observed in the detrusor of patients with invasive cancer, and was associated with the more severe OAB-like symptoms in these patients, whereas M3R downregulation was observed in the urothelium of these patients. These results indicated that the mechanisms leading to OAB-like symptoms may be distinct between $\mathrm{OAB}$ and bladder cancer.

M3R genes are conditional oncogenes (11) frequently expressed in digestive tract cancer types and considered to serve an important role in proliferation, differentiation, distant metastasis, transformation and carcinogenesis (22). They are considered to serve a key role in the proliferation and metastasis of cholangiocarcinoma (23), and their expression is influenced by the extent of differentiation, distant metastasis and site of cholangiocarcinoma. Whilst several studies have indicated that M3R upregulation is linked to cancer processes, others indicate that downregulation is involved $(9,11-13,22,23)$.

On one hand, M3R activation promotes proliferation, migration and angiogenesis in several types of cancer cells. It also promotes proliferation of normal urothelium and of the human urothelium-derived cell line UROtsa (24), and M3R-selective antagonists inhibit this effect. On the other hand, knocking down M3R increases proliferation of UROtsa (10). Transfecting bladder cancer cell lines such as T24 cells with an M3R expression plasmid significantly reduces proliferation (10). These data indicated that muscarinic M3R may serves a bidirectional regulatory role in normal urothelial proliferation (Fig. 4A), and that this regulatory mechanism is altered in bladder cancer urothelium (10-14) (Fig. 4B).

In the present cohort of patients, M3R appeared to act primarily as an anti-oncogene. M3R expression was significantly lower in invasive bladder cancer, compared with non-invasive cancer, and it was lower in the urothelium of invasive cancer, compared with tissue adjacent to the tumor. M3R expression increased with increasing distance from the tumor. In addition, M3R expression in urothelium negatively associated with PFS in patients with muscle-invasive cancer who underwent radical cystectomy.

M3R up-regulation promotes the proliferation of normal bladder detrusor cells (25), indicating that an observation of M3R expression increased in bladder cancer detrusor cells should be expected. It was determined that unlike in the urothelium, M3R expression in the detrusor was higher in the invasive group, compared with the non-invasive group. M3R has an established role as oncogene in colon and lung cancer; however, the present study demonstrated that it may be an anti-oncogene in urothelium of bladder cancer (7). The results indicated that M3R may serve different roles in the urothelium and in the detrusor of bladder cancer. Urgency maybe not just a secondary symptom due to detrusor invaded.

A previous study determined that mRNA levels of M3R were significantly upregulated only in low-grade tumor, 
A

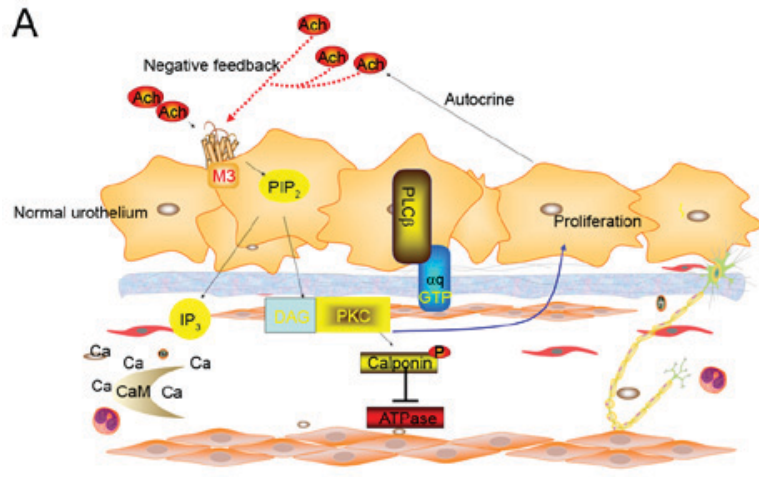

B

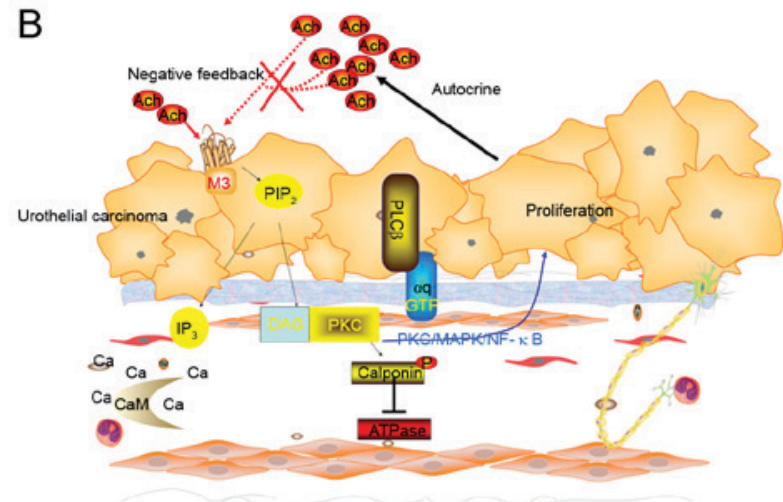

Figure 4. Signaling pathways of M3R regulates proliferation of urothelium (hypothesis based on previous reports). (A) It was speculated that a certain concentration of ACh combined to the M3R and stimulates proliferation of normal urothelium. urothelium release of ACh. The autocrined and paracrined ACh also combined to M3R. But M3R in turn inhibited urothelial proliferation when the concentration of ACh reaches a high level. The mechanism maintains the normal metabolism of urothelium. (B) It was speculated that the mechanism has changed in bladder cancer. ACh increased due to the autocrine and paracrine signals from the bladder urothelium and sub-urothelium tissues. However, the negative feedback of M3R on urothelial proliferation failed. The PKC pathway serves an important role in the regulation of urothelial proliferation of M3R according to the existed data. It is possible that the Ca ${ }^{2+} / \mathrm{DG}-\mathrm{PKC}$ pathway serves a major role in physiological while the $\mathrm{PKC} / \mathrm{MAPK} / \mathrm{NF}-\kappa \mathrm{B}$ pathway may serve a major role in bladder cancer cells. M3R, M1R, muscarinic cholinergic receptor 1 ; PKC/MAPK/NF- $\kappa \mathrm{B}$, protein kinase $\mathrm{C} /$ mitogen-activated protein kinase/nuclear factor- $\kappa \mathrm{B}$.

compared with normal biopsies tissue, and there were no difference in high-grade tumors (19). Similar to this study, the data from the present study also demonstrated that there were no differences between low-grade and high-grade tumors in M3R expression.

The results implicated $M 2 R$ as an anti-oncogene in bladder cancer. It was determined that $\mathrm{M} 2 \mathrm{R}$ expression was highest in tumor tissue and decreased with increasing distance from the tumor. It was also higher in the sub-urothelium of patients with invasive cancer. Among patients who underwent radical bladder cystectomy, M2R expression associated negatively with PFS. These results are consistent with study of Pacini et al (19) indicating that M2R helps regulate bladder cancer proliferation, migration and invasion.

Similar to M2R, the results indicate that M1R and M4R promote tumorigenesis and progression of bladder cancer. M1R in sub-urothelium was significantly higher in invasive cancer, and it was significantly lower in the urothelium of cancer tissue, compared with adjacent non-cancerous tissue. M1R urothelium and M4R sub-urothelial expression negatively associated with tumor T stage. And M4R in sub-urothelium was significantly higher in muscle-invasive cancer compared with non-muscle-invasive cancer. Therefore, it was considered that the M1R and M4R also serve an important role in tumorigenesis and progression of bladder cancer.

The association of AChE with patient survival and tumor aggressiveness had been confirmed in brain, lung, head and neck and hepatocellular cancer (26). A negative association was determined between ChE levels and PFS in patients with muscle-invasive cancer who underwent radical bladder cystectomy. Additionally, ACh interacts with M3R to stimulate malignant tumor growth in colon tumors (13). In addition, significantly lower proportions of ChAT-positive cells in the urothelium of muscle-invasive cancer were determined. This may indicate increased synthesis of ACh and/or reduced degradation of $\mathrm{ACh}$ in cancer tissue, leading to excessive accumulation of ACh, which promotes tumor cell proliferation $(13,27)$. Collectively, these observations indicated that the cholinergic signaling was over-stimulated in advanced bladder cancer. Cholinergic signaling may be a novel target to prevent and treat bladder tumor.

Higher expression of MMP-9 were observed in the sub-urothelium of muscle-invasive cancer and associated with PFS of patients who underwent radical cystectomy. These results are consistent with the hypothesis that MMP-9 acts as a downstream effector of cholinergic signaling in bladder cancer (28).

The results of the present study should be interpreted with caution due to several limitations. Although data were entered prospectively into the patient database, it cannot exclude that a number of patients with bladder cancer may have had $\mathrm{OAB}$; however, it is unlikely that the incidence of $\mathrm{OAB}$ was high enough to affect the results. A second limitation is that patients with OAB-like syndrome were diagnosed using subjective criteria, including urgency, urinary frequency and nocturia, which may be interfered with by drink water due to hematuria. Thirdly, completely normal human bladder urothelium in the experiments were not used as a control. Future studies should address these deficiencies in order to verify and extend the data. Additionally, preoperative clinical staging was performed in a number of patients, although pathological staging was applied to patients who underwent radical cystectomy.

The present study demonstrated that M3R expression was lower in invasive bladder cancer, but it was negatively associated with PFS. Another possible explanation is that M3R may serve a more complex biological behavior. Transforming growth factor- $\beta$ acts as a tumor suppressor normally, but also function as a tumor promoter in advanced cancers (29).

In conclusion, the present study indicated that cholinergic muscarinic signaling is altered in bladder cancer and is associated with prognosis. Tumorigenesis and development of bladder cancer appear to involve mAChRs, in particular a change in the regulatory role of M3R in the urothelium. M3R may act as an anti-oncogene in bladder cancer, unlike its oncogenic role in numerous other cancer types. 


\section{Acknowledgments}

Not applicable.

\section{Funding}

This work were supported by Hubei postdoctoral innovation fund (2016), Wuhan postdoctoral innovation fund (2016), the self-financing research of the Health Department of Guangxi Autonomous Region (grant nos. Z2016494 and Z2016499), Scientific research project in the Guangxi Zhuang Autonomous Region (grant no. KY2015LX060) and National Natural Science Foundation of China (grant no. 81560428).

\section{Availability of data and materials}

The datasets used and/or analyzed during the current study are available from the corresponding author on reasonable request.

\section{Authors' contributions}

WW and MW participated in the design of the study and performed the clinical analysis. XY and KX participated in study design and drafted the manuscript. LX, YL, QM, YT, HL, WY and QC participated in study design, literature search, and coordinating and performing the operation. All authors participated in the conception and design of the study, as well as data collection and interpretation, manuscript preparation and literature searches. All authors read and approved the final manuscript.

\section{Ethics approval and consent to participate}

Written informed consent was obtained from the patient for publication of this report and accompanying images.

\section{Consent for publication}

The patients provided written informed consent for publication.

\section{Competing interests}

The authors declare that they have no competing interests.

\section{References}

1. Siegel RL, Miller KD and Jemal A: Cancer statistics, 2018. CA Cancer J Clin 68: 7-30, 2018

2. Stenzl A, Cowan NC, De Santis M, Jakse G, Kuczyk MA, Merseburger AS, Ribal MJ, Sherif A and Witjes JA: The updated EAU guidelines on muscle-invasive and metastatic bladder cancer. Eur Urol 55: 815-825, 2009.

3. Babjuk M, Oosterlinck W, Sylvester R, Kaasinen E, Böhle A and Palou-Redorta J; European Association of Urology (EAU): EAU guidelines on non-muscle-invasive urothelial carcinoma of the bladder. Eur Urol 54: 303-314, 2008.

4. Kao LT, Huang CY, Lin HC and Chu CM: No increased risk of fracture in patients receiving antimuscarinics for overactive bladder syndrome: A retrospective cohort study. J Clin Pharmacol, Jan 9, 2018 (Epub ahead of print).

5. Balachandran AA and Duckett J: Metastatic breast cancer presenting as detrusor overactivity. BMJ Case Rep 2014: pii: bcr2014207920, 2014.
6. Haferkamp A, Schurch B, Reitz A, Krengel U, Grosse J, Kramer G, Schumacher S, Bastian PJ, Büttner R, Müller SC and Stöhrer M: Lack of ultrastructural detrusor changes following endoscopic injection of botulinum toxin type a in overactive neurogenic bladder. Eur Urol 46: 784-791, 2004.

7. Ito Y, Kashiwabara M, Yoshida A, Hikiyama E, Onoue S and Yamada S: Muscarinic receptor binding in rat bladder urothelium and detrusor muscle by intravesical solifenacin. Biol Pharm Bull 39: 1167-1171, 2016.

8. Hawthorn MH, Chapple CR, Cock M and Chess-Williams R: Urothelium-derived inhibitory factor(s) influences on detrusor muscle contractility in vitro. Br J Pharmacol 129: 416-419, 2000.

9. Wang CT, Chen TM, Mei CT, Chang CF, Liu LL, Chiu KH, Wu TM, Lan YC, Liu WS and Chen YH: The Functional Haplotypes of CHRM3 Modulate mRNA Expression and Associate with Bladder Cancer among a Chinese Han Population in Kaohsiung City. Biomed Res Int 2016: 4052846, 2016.

10. Sun $\mathrm{Y}$ and Chai T: 319 the role of $\mathrm{m} 3$ muscarinic signaling in bladder urothelial cancer cell proliferation and apoptosis. J Urol: $183,2010$.

11. Gutkind JS, Novotny EA, Brann MR and Robbins KC: Muscarinic acetylcholine receptor subtypes as agonist-dependent oncogenes. Proc Natl Acad Sci USA 88: 4703-4707, 1991.

12. Ashkenazi A, Ramachandran J and Capon DJ: Acetylcholine analogue stimulates DNA synthesis in brain-derived cells via specific muscarinic receptor subtypes. Nature 340: 146-150, 1989.

13. Von Rosenvinge EC and Raufman JP: Muscarinic receptor signaling in colon cancer. Cancers (Basel) 3: 971-981, 2011.

14. Parnell EA, Calleja-Macias IE, Kalantari M, Grando SA and Bernard HU: Muscarinic cholinergic signaling in cervical cancer cells affects cell motility via ERK1/2 signaling. Life Sci 91: 1093-1098, 2012

15. Homma Y, Yoshida M, Seki N, Yokoyama O, Kakizaki H, Gotoh M, Yamanishi T, Yamaguchi O, Takeda $M$ and Nishizawa O: Symptom assessment tool for overactive bladder syndrome-overactive bladder symptom score. Urology 68: 318-323, 2006

16. Yi X: Expression and clinical significance of M1-5 receptor subtype of bladder mucosa in patients with OAB [D]. Guang Zhou South Med Univ, 2011.

17. Humphrey PA, Moch H, Cubilla AL, Ulbright TM and Reuter VE: The 2016 WHO classification of tumours of the urinary system and male genital organs-part B: Prostate and bladder tumours. Eur Urol 70: 106-119, 2016.

18. Tully BT, Li M, Sun Y, Berkowitz J and Chai TC: Defects in muscarinic receptor cell signaling in bladder urothelial cancer cell lines. Urology 74: 467-473, 2009.

19. Pacini L, De Falco E, Di Bari M, Coccia A, Siciliano C, Ponti D, Pastore AL, Petrozza V, Carbone A, Tata AM and Calogero A: M2muscarinic receptors inhibit cell proliferation and migration in urothelial bladder cancer cells. Cancer Biol Ther 15: 1489-1498, 2014.

20. Bschleipfer T, Schukowski K, Weidner W, Grando S, Schwantes U, Kummer W and Lips K: Expression and distribution of cholinergic receptors in the human urothelium. Life Sci 80: 2303-2307, 2007.

21. Yamanishi T, Kaga K, Fuse M, Shibata C, Kamai T and Uchiyama $\mathrm{T}$ : The role of muscarinic receptor subtypes on carbachol-induced contraction of normal human detrusor and overactive detrusor associated with benign prostatic hyperplasia. J Pharmacol Sci 128: 65-70, 2015.

22. Park YS and Cho NJ: Enhanced proliferation of SNU-407 human colon cancer cells by muscarinic acetylcholine receptors. BMB Rep 41: 803-807, 2008.

23. Feng YJ, Zhang BY, Yao RY and Lu Y: Muscarinic acetylcholine receptor M3 in proliferation and perineural invasion of cholangiocarcinoma cells. Hepatobiliary Pancreat Dis Int 11: 418-423, 2012.

24. Arrighi N, Bodei S, Lucente A, Michel MC, Zani D, Simeone C, Cunico SC, Spano PF and Sigala S: Muscarinic receptors stimulate cell proliferation in the human urothelium-derived cell line UROtsa. Pharmacol Res 64: 420-425, 2011.

25. Arrighi N, Bodei S, Zani D, Michel MC, Simeone C, Cosciani Cunico S, Spano P and Sigala S: Different muscarinic receptor subtypes modulate proliferation of primary human detrusor smooth muscle cells via Akt/PI3K and map kinases. Pharmacol Res 74: 1-6, 2013.

26. Campoy FJ, Vidal CJ, Muñoz-Delgado E, Montenegro MF, Cabezas-Herrera J and Nieto-Cerón S: Cholinergic system and cell proliferation. Chem Biol Interact 259: 257-265, 2016. 
27. Spindel ER: Muscarinic receptor agonists and antagonists: Effects on cancer. Handb Exp Pharmacol 208: 451-468, 2012.

28. Peng Z, Heath J, Drachenberg C, Raufman JP and Xie G: Cholinergic muscarinic receptor activation augments murine intestinal epithelial cell proliferation and tumorigenesis. BMC Cancer 13: 204, 2013.
29. Liu WT, Huang KY, Lu MC, Huang HL, Chen CY, Cheng YL, Yu HC, Liu SQ, Lai NS and Huang HB: TGF- $\beta$ upregulates the translation of USP15 via the PI3K/AKT pathway to promote $\mathrm{p} 53$ stability. Oncogene 36: 2715-2723, 2017.

(c) (1) () This work is licensed under a Creative Commons Attribution-NonCommercial-NoDerivatives 4.0 International (CC BY-NC-ND 4.0) License. 